\title{
CRISPR-Cas9-mediated PD-1 and TCR Gene-deleted Anti-mesothelin CAR T-cells
}

\author{
National Cancer Institute
}

\section{Source}

National Cancer Institute. CRISPR-Cas9-mediated PD-1 and TCR Gene-deleted Antimesothelin CAR T-cells. NCI Thesaurus. Code C158606.

A preparation of human T-lymphocytes transduced with a chimeric antigen receptor (CAR) specific for the tumor-associated antigen (TAA) mesothelin and gene-edited with the clustered regularly interspaced short palindromic repeats (CRISPR)-Cas9 nuclease complex to eliminate endogenous TCR and programmed death 1 (PD-1; PDCD1; CD279; programmed cell death-1) expression, with potential immunostimulating and antineoplastic activities. The CRISPR guide RNA (gRNA) specifically targets and binds to complementary sites on TCRalpha, TCRbeta and PD-1. In turn, Cas9 cleaves these specific DNA sites, thereby disrupting transcription. Upon isolation, transduction, electroporation with TCRalpha, TCRbeta and PD-1 gRNAs, which are complexed to Cas9 RNA to disrupt expression of endogenous TCRalpha, TCRbeta and PD-1, expansion ex vivo, and introduction into the patient, the CRISPR-Cas9-mediated PD-1 and TCR genedeleted anti-mesothelin CAR T-cells recognize and bind to mesothelin-overexpressing tumor cells. This may result in a specific cytotoxic T-lymphocyte (CT L)-mediated killing of mesothelin-positive tumor cells. PD-1, an immune checkpoint receptor expressed on Tcells, plays a key role in tumor immune evasion by binding to its ligand programmed death ligand 1 (PD-L1; cluster of differentiation 274; CD274; programmed cell death-1 ligand 1) expressed on tumor cells. By removing PD-1 from T-cells, PD-1-mediated signaling is halted which may decrease $\mathrm{T}$-cell exhaustion and may enhance $\mathrm{T}$-cell activity against the mesothelin-expressing tumor cells. Removal of endogenous TCR reduces TCR competition for expression, increases the persistence and function of the expressed transgenic TCR, enhances resistance to T-cell exhaustion and increases T-cell activity. Mesothelin is upregulated on a variety of tumor cell types. 